\title{
The Participation of Pyridoxal Phosphate, Carnosine and Calcium in the Reversal of Contraction in Muscle Fiber Systems ${ }^{1}$
}

\author{
D. E. S. BROWN, E. B. CAREW AND R. S. BALER \\ From the Department of Zoology, University of Michigan, Ann Arbor, Michigan
}

Received August 6, 1962

\begin{abstract}
A relaxing system requiring phosphocreatine as the relaxant may be restored to glycerinated psoas fibers by incubation in a reaction mixture containing pyridoxal phosphate, calcium, carnosine, magnesium, ATP, and sodium maleate at $\mathrm{pH} 6.5$. The capacity to relax, once established, persists after elution in buffer of the diffusible fraction of the reactants. The relaxation by phosphocreatine is reversed by calcium. The restored relaxing system is also subject to relaxation by the dialyzable fraction of the microsomal-free crude muscle extract, but in this case carnosine is not required in the reaction medium.
\end{abstract}

\section{INTRODUCTION}

Kaldor and Gergely (1) observed that pyridoxal phosphate, ${ }^{2}$ in relatively large concentrations, inhibited ATPase activity and syneresis of myofibrils and that the effect is reversed by carnosine. In single glycerinated fibers, Briggs, Kaldor, and Gergely (2) found that carnosine reversed the relaxation induced by crude muscle extract or the reconstituted relaxing system. In recent studies in our laboratories of methods of restoring a relaxing factor system in wellwashed glycerinated muscle, it was observed that treatment with micromolar concentrations of PLP and carnosine was effective, provided micromolar amounts of calcium were present. Although the combination of PLP, calcium, and carnosine did not cause relaxation, the fiber became susceptible to

1 This work was aided by a grant from the Muscular Dystrophy Associations of America, Ine.

2 Abbreviations: SM, standard medium, $5 \mathrm{mM}$ $\mathrm{ATP}, 4 \mathrm{~m} M \mathrm{Mg}^{++}, 0.06 \mathrm{M}$ sodium maleate, $\mathrm{pH}$ 6.8; DFR, defined factors for relaxation, PLP plus $\mathrm{Ca}^{++}$plus carnosine; DRS, defined relaxing system; CP, phosphocreatine; PLP, pyridoxal phosphate; ATP, adenosine triphosphate; ATPase adenosinetriphosphatase; EDTA, ethylenediamine tetraacetate. relaxation by CP. Fibers treated with only PLP and calcium failed to relax on the addition of $\mathrm{CP}$, but relaxation resulted when the dialyzable fraction of a muscle extract free of microsomes was substituted for $\mathrm{CP}$. The present paper presents experiments demonstrating the properties of this defined relaxing system.

\section{MATERIALS AND METHODS}

Psoas fiber bundles were removed from an anesthetized rabbit and extracted in $50 \%$ glycerol with $0.125 M$ histidine buffer at $\mathrm{pH} 6.4$. Extraction was carried out in a roto-tube apparatus at $0^{\circ}$ for 3 days, the extraction medium being changed every $8 \mathrm{hr}$. After $24 \mathrm{hr}$, extraction, the fiber bundles were subdivided into bundles about 0.5 $\mathrm{mm}$. in diameter. The fibers were stored at $-2^{\circ}$ to $-4^{\circ}$ and experimented upon after $1-120$ days of storage.

In performing an experiment, a group of two to four muscle fibers was mounted vertically at rest length on an isometric lever and immersed in $0.125 M$ maleate buffer containing $4 \mathrm{~m} M$ EDTA, at pH 6.4 and $20^{\circ} \mathrm{C}$., for $60 \mathrm{~min}$. The medium was then replaced by the buffer alone, to elute the EDTA. After $15 \mathrm{~min}$, the fibers were placed in the standard reaction mixture $S M$, consisting of $5 \mathrm{~m} M$ ATP, $4 \mathrm{~m} M \mathrm{MgCl}_{2}$, and $0.06 M$ maleate buffer, pH 5.2. On immersion, the fiber contracts and then relaxes to a low steady level of tension. The experimental contractions were induced 
either by increasing the $\mathrm{pH}$ abruptly from $\mathrm{pH}$ 5.2 to $\mathrm{pH} 6.8$ or gradually in $0.1 \mathrm{pH}$ units by the addition of small amounts of $1 \mathrm{~N} \mathrm{NaOH} .^{3}$ The substances to be tested were added either prior to or after the development of maximum tension.

In plotting the data, tension $Y$ is expressed as a fraction of the maximum tension $Y_{0}$ achieved, i.e., $Y / Y_{0}$.

The $\mathrm{pH}$ was measured by a Beckman model 9600 instrument with glass electrodes, No. 39166, mounted permanently in the reaction chamber.

The temperature was controlled to $\pm 0.1^{\circ}$, by Dewar flask temperature baths in which the reaction vessel was immersed, and measured continuously by a thermocouple lying alongside the muscle fiber. Stirring of the reaction medium was maintained by a continuous stream of air bubbles.

The dialyzable cofactor from fresh muscle was prepared according to Briggs, Kaldor, and Gergely (2), involving $2 \mathrm{hr}$. centrifugation at $35,000 \times g$ to remove the microsomal fraction, followed by dialysis at $20^{\circ}$ against $0.125 \mathrm{M}$ maleate buffer at $\mathrm{pH} 6.4$ for $24 \mathrm{hr}$. The dialyzate was used in a dilution 1:10 with respect to the crude extract, and the mixture was adjusted to a concentration of $0.06 \mathrm{M}$ maleate buffer at $\mathrm{pH} 6.8$.

Crystalline disodium ATP was obtained from Pabst Research Biochemicals, St. Louis, Mo; CP from the Sigma Chemical Co., St. Louis, Mo.; PLP and L-carnosine from the California Corporation for Biochemical Research, Los Angeles, Cal.

\section{RESULTS}

\section{'The Action of Plu, Calcium and Carnosine in Restoring a Relaxing System}

When glycerinated fibers are prepared as described, CP does not cause relaxation, but this response may be induced by treating the fiber with a watery extract of freshly minced muscle (3).

In such well-extracted fibers relaxation may be induced by adding a combination of $80 \mu M$ PLP, $0.6 \mu M \mathrm{CaCl}_{2}, 80 \mu M$ carnosine (constituting the DFR), and $10 \mathrm{~m} M \mathrm{CP}$ to a fiber already contracted in the standard re-

\footnotetext{
${ }^{3}$ When handled in this way, the fibers develop full tension at rest length without the usual breakage which results when fibers are transferred directly from the buffer medium to the $\mathrm{SM}$ at $\mathrm{pH} 6.8$.

4 The effective range of concentration determined for these substances is $10-80 \mu M$ for PLP, $0.1-0.6 \mu M$ for $\mathrm{Ca}^{++}$, and $0.01-1.0 \mathrm{~m} M$ for carnosine.
}

action mixture at $\mathrm{pH} 6.8$ (Fig. $1 A){ }^{4}$ The relaxation is reversed on the addition of $0.1-1$ $\mathrm{m} M \mathrm{Ca}^{++}$. If PLP, calcium, carnosine, or CP is lacking, relaxation does not occur. Moreover, pyridoxal cannot be substituted for PLP.

If the fiber is treated before contraction with PLP, calcium, and carnosine, tension develops as in the control, and relaxation ensues when CP is added (Fig. $1 B$ ). When PLP, calcium, and CP are present initially in the medium, tension develops as before but the addition of carnosine does not cause relaxation.

A unique situation obtains with respcct to calcium, for if the muscle is contracted in SM, PLP, and carnosine, and then calcium chloride is added to a final concentration of $0.6 \mu M$, the fiber is not relaxed by CP. Evidently PLP and calcium must first participate in some specific reaction within the fiber if the addition of carnosine is to confer a relaxing capacity with respect to $\mathrm{CP}$.

\section{Persistexce of the Relaxing System}

The defined relaxing system, DRS, once established, persists after the free PLP carnosine, and calcium are removed. This may be shown by developing the relaxing system by immersing the fiber in the reaction mixture, SM, calcium, PLP, and carnosine at $\mathrm{pH}$ 6.5. When the maximum tension is developed, the fibers are immersed in $50 \%$ glycerol-histidine at $\mathrm{pH} 6.4,0^{\circ} \mathrm{C}$, and left for at least $72 \mathrm{hr}$. without stirring. On returning the fibers to $\mathrm{SM}$ at $\mathrm{pH} 6.8$, full tension develops and relaxation ensues on the addition of $10 \mathrm{~m} M \mathrm{CP}$.

In fibers where the relaxing system has been restored in the above manner, the addition of calcium is not necessary for relaxation by CP. Nevertheless, calcium is involved since treatment with $4 \mathrm{~m} M$ EDTA prior to contraction followed by its elution eliminates the CP effect. However, the usual relaxation by $\mathrm{CP}$ returns if the fiber is contracted in $\mathrm{SM}+0.6 \mu M \mathrm{Ca}^{++}$.

The persistence of the DRS makes possible the determination of the $\mathrm{pH}$ range in which the DRS is set up, and also the importance of ATP and magnesium in the restoration of the relaxing system. 

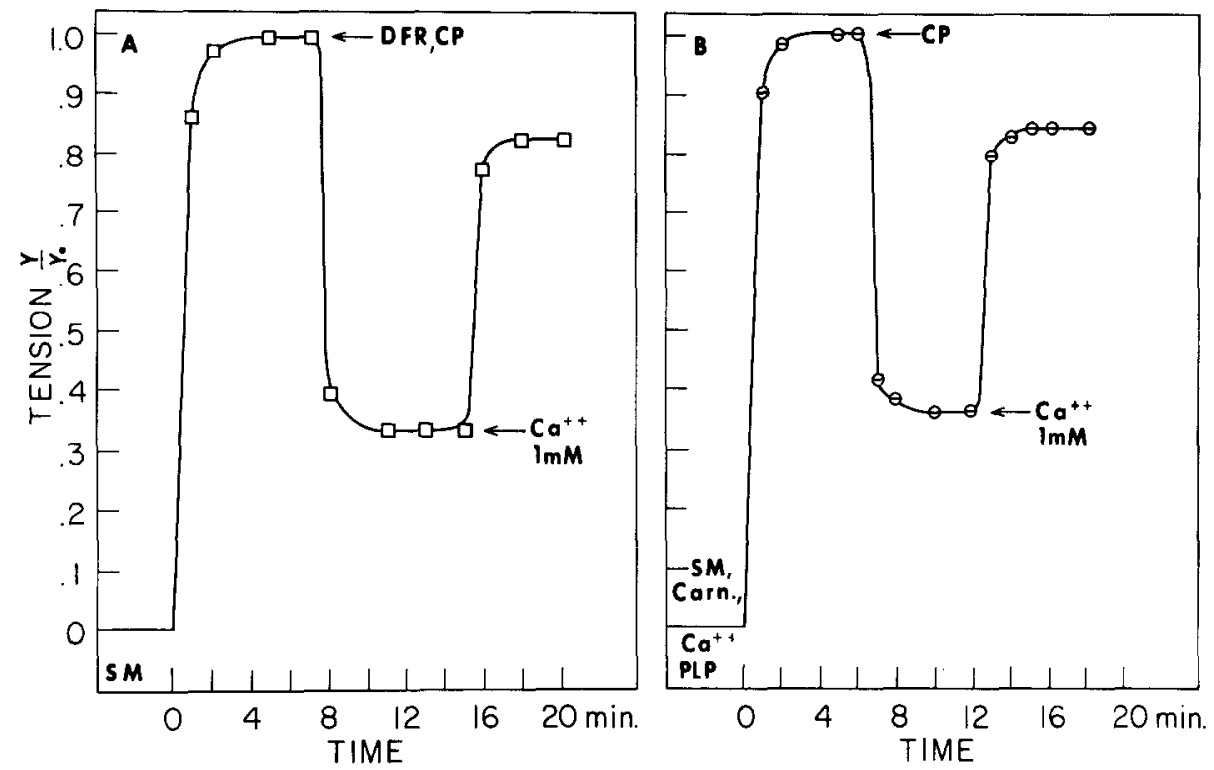

Fig. 1. A. Contraction in SM at $\mathrm{pH} 6.8$, with relaxation induced by addition of DFR plus CP. Contraction reversed by $\mathrm{Ca}^{++}$. Tension $Y$ expressed as fraction of the maximum tension $Y_{n} . B$. Contraction in SM plus DFR at pH 6.8. Relaxation induced by addition of $\mathrm{CP}$ and its reversal by $\mathrm{Ca}^{++}$.

In a serics of experiments, fibers were immersed in the reaction mixture, SM plus calcium, PLP, and carnosine, at specific $\mathrm{pH}$ values in the range 5.2-6.6 and left until the maximum tension developed. The fiber was then immersed for $1 \mathrm{hr}$. in buffer alone at the same $\mathrm{pH}$ to elute the diffusible fraction of the reaction mixture. At the end of this period the fiber was contracted again in the standard reaction mixture at $\mathrm{pH} 6.8$. When the maximum tension was attained, $\mathrm{CP}$ was added to test for the presence of the DRS. The amount of DRS set up, expressed as the fractional relaxation produced by $\mathrm{CP}$, increases with $\mathrm{pH}$ in the range from $\mathrm{pH} 5.4$ to 6.7 with half-DRS at $\mathrm{pH} 5.9$. In similar experiments in which $\Lambda \mathrm{TP}$ and magnesium were absent during incubation, no relaxing system was developed. In view of this situation, the development of the DRS is always accompanied by the development of tension, but this does not necessarily mean that tension per se is essential to setting up the DRS. A more reasonable interpretation would be that chemical events involving ATP and magnesium are participating in the formation of the stable relaxing complex as well as in contraction.

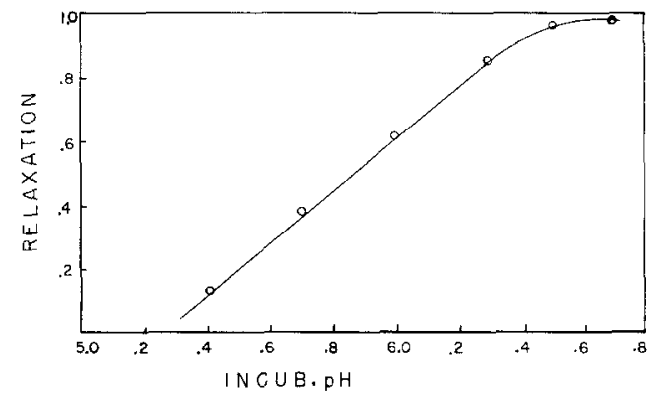

Fis. 2. Fractional relaxation, $\left(Y_{0}-Y\right) / Y_{0}$, produced by $10 \mathrm{~m} M \mathrm{CP}$ at $\mathrm{pH} 6.8$ following incubation at $0^{\circ} \mathrm{C}$. in $\mathrm{SM}$ plus DFR at a series of pH's as indicated. Fibers washed for $1 \mathrm{hr}$. in $50 \%$ glycerol-histidine buffer $\mathrm{pH} 6.4$ before testing. Each point on the graph refers to a different fiber.

The $\mathrm{pH}$ dependence of the DRS (Fig. 2) suggests the $\mathrm{pH}$ dissociation of some myofibrillar component, the relaxing system then resulting from interaction of the DFR with either the dissociated or associated component to form the $\mathrm{pH}$-stable relaxing complex.

\section{Relaxation by the Dialyzate of Muscle Extract}

Gergely, Kaldor, and Briggs (4) have shown that the dialyzate of a crude muscle 
extract freed of microsomes contains a dialyzable cofactor which in the presence of microsomes causes relaxation. Accordingly, experiments were performed to test the dialyzable cofactor in relation to the DRS. As shown in Fig. $3 A$, a fiber contracted at $\mathrm{pH}$ 6.8 in SM relaxes on the addition of the dialyzable fraction plus PLP and calcium, but carnosine is not required. In the absence of PLP or calcium, the dialyzate has no effect. If the fiber is contracted in SM, PLP, and $0.6 \mu M \mathrm{Ca}^{++}$(Fig. $3 B$ ), relaxation ensues on the addition of the dialyzable fraction, even when $2 \mathrm{~m} M$ potassium oxalate is present. When the fiber is contracted in SM and the agents are then added in sequence, the dialyzable fraction causes relaxation only if applied after the addition of PLP and calcium. In comparison with the DFR-CP system, it may be noted that the dialyzable fraction acts analogously to the combination of CP and carnosine, while PLP plus calcium duplicates the action of such microsomal fractions as require the dialyzable fraction to cause relaxation.

\section{pH Axd the Action of DFR AND CP ON TENSION}

As Goodall and Szent-Györgyi (3) have shown, the contraction and relaxation of glycerinated fibers containing a CP-relaxing system may be brought about by increasing and decreasing the $\mathrm{pH}$. The application of this procedure in the current studies brings out certain relationships between the DFR and the $\mathrm{pH}$-tension curve.

When the fiber is immersed in either SM plus $0.6 \mu M \mathrm{Ca}^{++}$, or SM plus DFR at $\mathrm{pH}$ 5.2 , it contracts and then relaxes (Fig. $4 A$ ). On increasing the $\mathrm{pH}$, tension develops, reaching half-tension within $0.3 \mathrm{pH}$ unit and the maximum value at $\mathrm{pH}$ 6.1. At higher $\mathrm{pH}$ values the maximum is sustained. As shown in the preceding section, the DRS is being set up over this $\mathrm{pH}$ range, but it is evident that its establishment does not affect the $\mathrm{pH}$ tension relation. When $\mathrm{CP}$ is added at $\mathrm{pH}$ 6.8 the fiber relaxes but thereafter exhibits a new $\mathrm{pH}$-tension relation; thus a further increase in $\mathrm{pH}$ leads to a redevelopment of tension, while a decrease in $\mathrm{pH}$ causes the fiber to relax, approaching zero tension at about $\mathrm{pH}$ 6.2. The $\mathrm{pH}$ tension relation thereafter remains set in the $\mathrm{pH}$ range $6.2-7.4$ (Fig. $4 B$ ), and a contraction-relaxation cycle may be produced several times by simply raising and lowering the $\mathrm{pH}$.

The transition to the CP type system is shown to advantage in Fig. $4 B$, where both DFR and CP are present from the start of
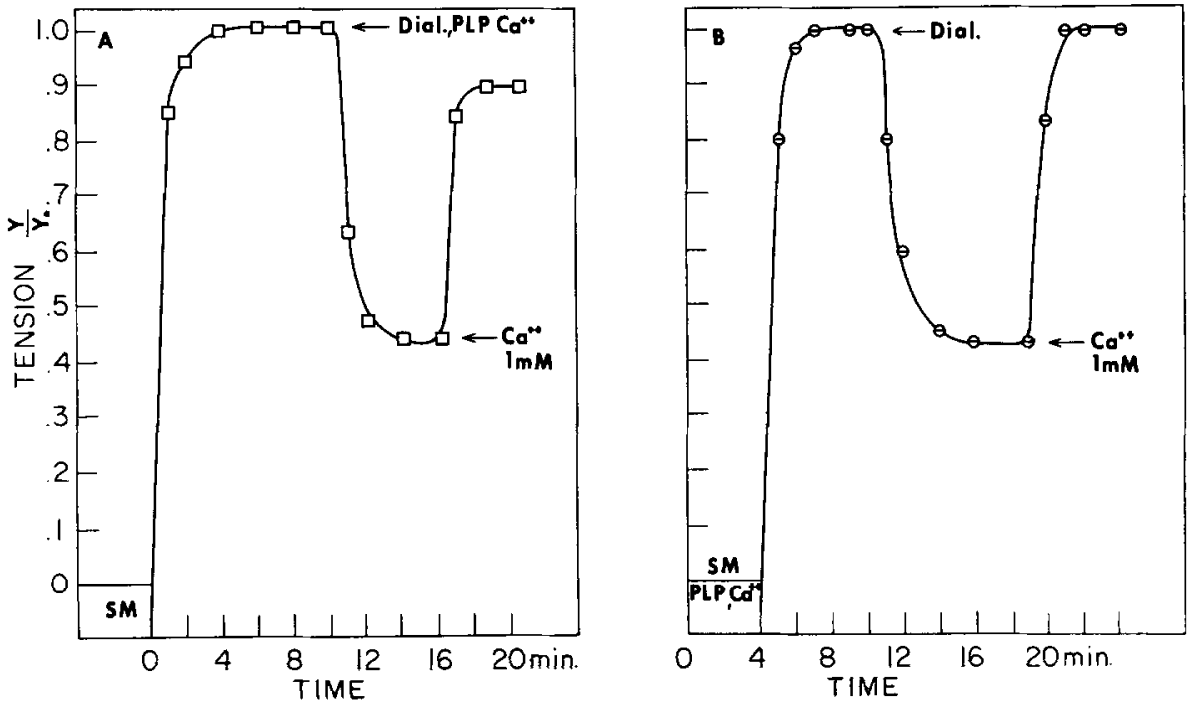

FIG. 3. A. Contraction in SM at pH 6.8. Relaxation induced by addition of dialyzable cofactor, PLP, and $\mathrm{Ca}^{++}$, and its reversal by $1 \mathrm{~m} M \mathrm{Ca}^{++} . B$. Contraction in SM, PLP, and $\mathrm{Ca}^{++}$. Relaxation induced by dialyzable cofactor and its reversal by $1 \mathrm{mM} \mathrm{Ca} \mathrm{Ca}^{++}$. 

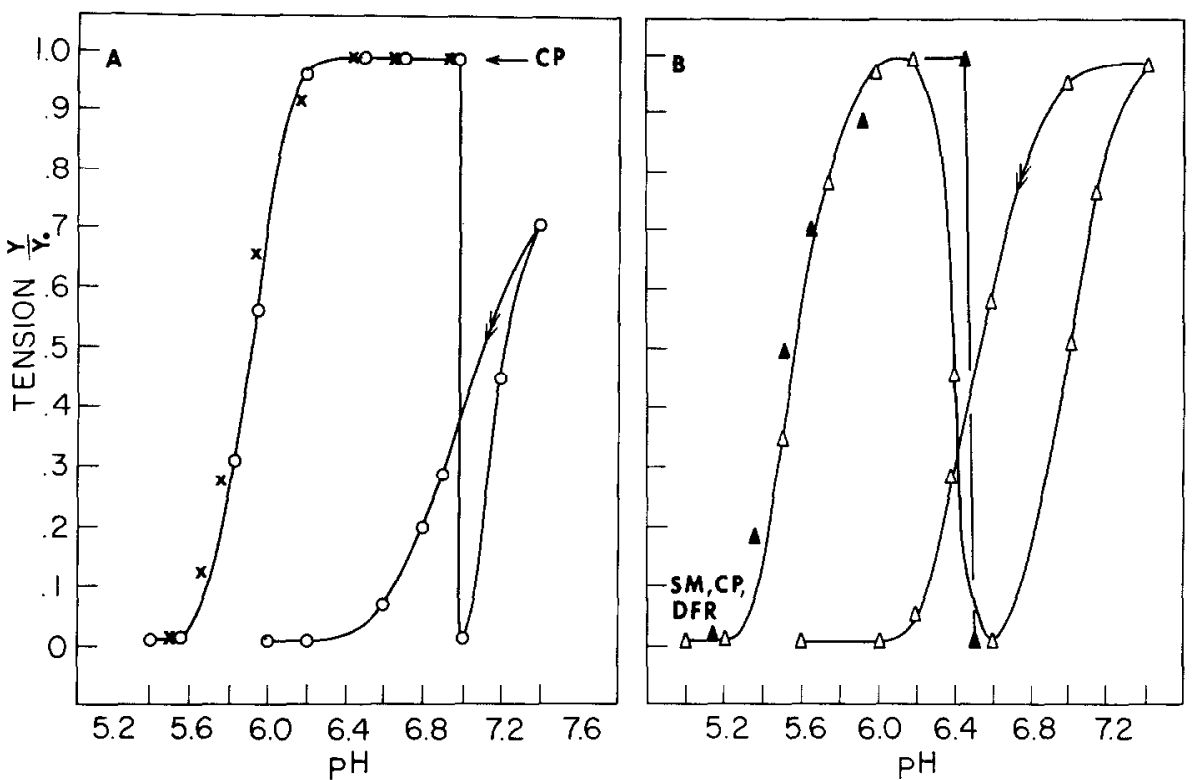

Fig. 4. A. $\times$, tension-pH curve in SM plus $0.6 \mu M \mathrm{Ca}^{++} ; \bigcirc$, tension-pH curve in SM plus DFR and relaxation induced at arrow by CP. Redevelopment of tension induced by further increasing $\mathrm{pH}$ and relaxation by lowering $\mathrm{pH} . B . \triangle$, tension- $\mathrm{pH}$ curve developed in SM, DFR, and $10 \mathrm{~m} M \mathrm{CP}$. Relaxation ensues at $\mathrm{pH} 6.2$, reaching a minimum at $\mathrm{pH} 6.8$. With further increase in $\mathrm{pH}$, tension redevelops to a maximum. Relaxation accompanies reduetion in $\mathrm{pH}$. O, tension-pH curve developed in SM, DFR, and $20 \mathrm{~m} M \mathrm{CP}$. Relaxation occurs abruptly at $\mathrm{pH} 6.8$.

the experiment. In this case, where the relaxing system is being set up in the presence of $\mathrm{CP}$, relaxation does not set in until $\mathrm{pH} 6.4$ with $10 \mathrm{~m} M$ CP and $\mathrm{pH} 6.7$ with $20 \mathrm{~m} M$ CP. In the latter concentration the fiber is fully contracted at $\mathrm{pH} 6.7$ and completely relaxed at $\mathrm{pH} 6.8$. The increment in $\mathrm{pH}$ required to place the CP-relaxing system in control of contractile events is exceptionally small and raises the possibility that the onset of relaxation in normal muscle might be initiated in a similarly abrupt fashion by some chemical event in contraction.

\section{DISCUSSION}

The foregoing results show that the capacity to be relaxed by $\mathrm{CP}$ may be restored to glycerinated psoas fibers by incubation at $\mathrm{pH} 6.6$ in a medium consisting of ATP, magnesium, PLP, calcium, carnosine, and sodium maleate buffer. If the agents are added sequentially, PLP and calcium must precede carnosine for $\mathrm{CP}$ to produce relaxation. This seems to indicate that the incorporation of PLP and calcium at certain loci is necessary for carnosine to interact with the system. The fact that the capacity to relax, once established, remains after the elution of the diffusible fraction of PLP, calcium, and carnosine indicates the formation of a relatively stable relaxable complex. Once this is established, the ATP-contraction system becomes subject to relaxation by CP.

The implications of these findings in reference to current knowledge of relaxing factor systems are several. It is generally considered (5-8) that the relaxation of the ATPaseactomyosin contraction is brought about by a joint action of the myofibrils and granules immediately in contact with them. Of the granules, the heavier ones which include mitochondria appear to have a higher relaxing activity than the microsomes. The microsomal contribution to relaxation is known to depend on reactions involving, among other things, a magnesium-activated ATPase, myokinase, a creatine transphorylase, and, as Molnar and Lorand (9) have recently shown, a phosphate acceptor 
with CP as donor. From the microsomalfree, fresh muscle extract a dialyzable cofactor is obtained which, jointly with the microsomal fraction, constitutes a complete relaxing system. Under certain conditions a microsomal-free, fresh muscle extract per se causes relaxation, thus indicating the presence of a soluble relaxing factor (10). The reversal of syneresis by this substance is accompanied by an inhibition of the myofibrillar ATPase.

On the basis of the action of PLP in producing a syneresis of the myofibrils, Kaldor and Gergely (1) proposed that it may be a model of the hypothetical relaxing substance produced by the microsomes plus cofactor. Exploring further the possible ways that such a hypothetical relaxing substance could cause relaxation, these investigators showed that in myofibrils the reversal of syneresis by PLP was accompanied by an inhibition of ATPase, and that both actions were reversed by carnosine. In addition, PLP was shown to inhibit appreciably the ATPase of reconstituted actomyosin and to a lesser extent myosin. Neither of these effects was reversed by carnosine, although the latter inhibited myosin ATPase to a small degree. Clearly PLP and carnosine exert an action directly on the ATPase-actomyosin system.

The question naturally arises whether PLP, calcium, and carnosine are serving to restore the ability of the granules to produce the hypothetical relaxing substance or whether certain of these factors also act directly on the myofibril.

As Kaldor and Gergely pointed out (1), the concentration of PLP and carnosinc needed in their studies was unreasonably high on physiological standards. In the present studies, however, the effective concentrations are so low and act under such specific conditions that their involvement under physiological conditions seems possible.
A unique property of the defined factor system is that the reaction of the substances with the fiber proceeds only in the presence of ATP and magnesium, and further that the irreversible relaxable complex is set up only when the three substances-PLP, calcium, and carnosine-are present. This suggests a chain of reactions involving the splitting of ATP and the complexing of the other agents at its expense. A factor in the reaction system may be the action of PLP as a coenzyme or the well-known property of PLP first to chelate cations and then to form bridges with carnosine and other peptidcs. The present evidence points toward the possibility that such reactions may directly involve the myofibril as well as the granules.

\section{ACKNOWLEDGMENT}

We wish to express our appreciation to Dr. Karl F. Guthe for his valuable counsel during the course of these studies.

\section{REFERENCFS}

1. Kaldor, G., and Gergeis, J., Arch. Biochem. Biophys. 80, 393 (1959).

2. Briggs, F. N., Kaldor, G., and Gergely, J., Biochim. et Biophys. Acta 34, 211 (1959).

3. Goodall, M. C., and Szent-Györgyi, A. G., Nature 172, 84 (1953).

4. Gergely, J., Kaldor, G., and Briggs, F. N., Biochim. et Biophys. Acta 34, 218 (1959).

5. Perry, S. V., Ann. Rev. Biochem. 30, 473 (1961).

6. Lorand, L., and Molnar, J., in "Muscle as a Tissue." (K. Rodahl and S. M. Horvath, eds.), p. 97. MeGraw-Hill, New York, 1962.

7. Weber, H. H., Ann. N. Y. Acad. Sci. 81, (2), 409 (1959).

8. Gerefiy, J., Ann. N. Y. Acad. Sci. 81, (2), 490 (1959).

9. Molnar, J., and Lorand, L., Arch. Biochem. Biophys. 98, 356 (1962).

10. Fuchs, F., AND Briggs, F. H., Biochim. et Biophys. Acta 51, 433 (1961). 